OPEN ACCESS

Edited by:

Tim Vanmierlo,

University of Hasselt, Belgium

Reviewed by:

Hemmen Sabir

Essen University Hospital, Germany

Thomas Ragnar Wood,

University of Washington,

United States

${ }^{*}$ Correspondence:

Mariya Hristova

m.hristova@ucl.ac.uk

Specialty section:

This article was submitted to

Multiple Sclerosis and

Neuroimmunology,

a section of the journal

Frontiers in Immunology

Received: 31 March 2019 Accepted: 21 October 2019 Published: 29 November 2019

Citation:

Sisa C, Agha-Shah Q, Sanghera B, Carno A, Stover $C$ and Hristova $M$ (2019) Properdin: A Novel Target for Neuroprotection in Neonatal Hypoxic-Ischemic Brain Injury. Front. Immunol. 10:2610 doi: 10.3389/fimmu.2019.02610

\section{Properdin: A Novel Target for Neuroprotection in Neonatal Hypoxic-Ischemic Brain Injury}

\author{
Claudia Sisa ${ }^{1}$, Qudsiyah Agha-Shah ${ }^{1}$, Balpreet Sanghera ${ }^{1}$, Ariela Carno ${ }^{1}$, Cordula Stover ${ }^{2}$ \\ and Mariya Hristova ${ }^{1 *}$ \\ ${ }^{1}$ Perinatal Brain Repair Group, UCL Institute for Women's Health, Maternal \& Fetal Medicine, London, United Kingdom, \\ ${ }^{2}$ Department of Infection, Immunity and Inflammation, University of Leicester, Leicester, United Kingdom
}

Background: Hypoxic-ischemic $(\mathrm{HI})$ encephalopathy is a major cause of neonatal mortality and morbidity, with a global incidence of 3 per 1,000 live births. Intrauterine or perinatal complications, including maternal infection, constitute a major risk for the development of neonatal $\mathrm{HI}$ brain damage. During $\mathrm{HI}$, inflammatory response and oxidative stress occur, causing subsequent cell death. The presence of an infection sensitizes the neonatal brain, making it more vulnerable to the $\mathrm{HI}$ damage. Currently, therapeutic hypothermia is the only clinically approved treatment available for $\mathrm{HI}$ encephalopathy, however it is only partially effective in $\mathrm{HI}$ alone and its application in infection-sensitized $\mathrm{HI}$ is debatable. Therefore, there is an unmet clinical need for the development of novel therapeutic interventions for the treatment of HI. Such an alternative is targeting the complement system. Properdin, which is involved in stabilization of the alternative pathway convertases, is the only known positive regulator of alternative complement activation. Absence of the classical pathway in the neonatal $\mathrm{HI}$ brain is neuroprotective. However, there is a paucity of data on the participation of the alternative pathway and in particular the role of properdin in $\mathrm{HI}$ brain damage.

Objectives: Our study aimed to validate the effect of global properdin deletion in two mouse models: $\mathrm{HI}$ alone and LPS-sensitized HI, thus addressing two different clinical scenarios.

Results: Our results indicate that global properdin deletion in a Rice-Vannucci model of neonatal $\mathrm{HI}$ and LPS-sensitized HI brain damage, in the short term, clearly reduced forebrain cell death and microglial activation, as well as tissue loss. In $\mathrm{HI}$ alone, deletion of properdin reduced TUNEL+ cell death and microglial post-HI response at $48 \mathrm{~h}$ post insult. Under the conditions of LPS-sensitized HI, properdin deletion diminished TUNEL+ cell death, tissue loss and microglial activation at $48 \mathrm{~h}$ post- $\mathrm{HI}$.

Conclusion: Overall, our data suggests a critical role for properdin, and possibly also a contribution in neonatal $\mathrm{HI}$ alone and in infection-sensitized $\mathrm{Hl}$ brain damage. Thus, properdin can be considered a novel target for treatment of neonatal $\mathrm{HI}$ brain damage.

Keywords: properdin, complement, alternative pathway, hypoxia, ischemia, neuroprotection, neonate, infection 


\section{INTRODUCTION}

Oxygen deprivation around the time of birth is a major cause of neonatal hypoxic ischemic (HI) brain damage affecting 1-3 per 1,000 live births in developed countries and increasing to 26 per 1,000 in the developing world (1). Of the affected neonates, 15$25 \%$ die during the neonatal period and $25 \%$ of the survivors develop neurological sequelae such as epilepsy, cerebral palsy and cognitive defects (2), resulting in significant psychological and socioeconomic burden on the patient, family and healthcare system (1).

The pathophysiology of HI brain damage involves inflammation, oxidative stress, excito-toxicity and cell death (3-6).

Pre-exposure of the preterm infant to a bacterial infection sensitizes the brain, making it more susceptible to the $\mathrm{HI}$ insult. Bacterial lipopolysaccharide (LPS) - the major component of the outer membrane of most Gram-negative bacteria-is a strong immune stimulator and enhances cerebral damage and lesions in HI brain injury $(7,8)$.

Therapeutic hypothermia $(\mathrm{TH})$ is the standard clinical care for moderate to severe $\mathrm{HI}$ injury, however it is effective in only $55 \%$ of cases, while the remaining $45 \%$ of treated infants still develop neurological deficits (9). Thus, further studies on improving the success of $\mathrm{TH}$ and finding therapeutic alternatives are urgently required.

The effect of $\mathrm{TH}$ in infection-sensitized HI conditions is pathogen dependent (10). In a rat model of LPS-sensitized $\mathrm{HI}$, TH failed to reduce mortality and tissue damage (11). In clinical studies looking at the effect of TH on neonates exposed to intrauterine infection, $\mathrm{TH}$ treatment did not counteract inflammation (12).

The lack of effect of TH in LPS-sensitized HI could be attributed to inter-individual variability (7). Additionally, body cooling following $\mathrm{HI}$ alone is suggested to be immunosuppressive $(13,14)$, therefore counteracting the physiological attempt of the immune system in fighting bacterial infection.

The inability of TH to protect the neonatal brain in LPSsensitized HI, and its limited outcome in treatment of $\mathrm{HI}$ alone, support the urge to investigate new therapeutic alternatives or augmentation strategies for $\mathrm{TH}$.

Such an alternative is targeting the complement system, a cascade of over 30 proteins critically involved in innate immunity. The activated complement promotes inflammation and anaphylatoxin release and comprises three pathwaysclassical, lectin, and alternative. While the classical pathway (CP) is mainly activated by external pathogens, the alternative one (AP) is spontaneously active and also amplifies the other two pathways (15). Properdin is a plasma glycoprotein released mainly by leukocytes in response to pro-inflammatory stimuli (16). It is the only known positive regulator of the AP; in fact, properdin facilitates the constitutively active AP either by stabilizing the $\mathrm{C} 3$ convertase $\mathrm{C} 3 \mathrm{bBb}$ or by binding to susceptible surfaces, thus serving as a platform for de novo $\mathrm{C} 3 \mathrm{bBb}$ assembly $(17,18)$. This causes opsonization of target molecules through C3b and further activation of the complement cascade, culminating in the formation of the membrane attack complex (C5b-C9).

Clinical data associates neonatal HI with depleted C3 expression (19) and increased serum levels of C3a and C5a following fetal acidosis (20). While the role of properdin in inflammation has been widely studied $(21,22)$, there is a paucity of data surrounding the role of properdin in neonatal HI. It could be speculated that $\mathrm{HI}$ upregulates properdin levels and leads to increased anaphylatoxin production and pro-inflammatory activation of microglia and astrocytes. This study aims to elucidate the role of properdin in neonatal $\mathrm{HI}$ alone and in LPS-sensitized HI in the short term. Our data demonstrate the neuroprotective effect of global properdin deletion in both $\mathrm{HI}$ alone and LPS-sensitized HI at $48 \mathrm{~h}$ post-HI, suggesting this complement regulator as an attractive therapeutic target in neonatal HI and LPS-sensitized HI.

\section{MATERIALS AND METHODS}

\section{Animal Use}

Properdin-deficient mice were generated by site-specific genetic engineering, rendering mice deficient of the serum protein properdin and thereby lacking the amplification loop of complement activation (23). They have been maintained by crossing heterozygous properdin deficient female mice with wild type male C57Bl/6 mice and were obtained from the University of Leicester. Genotyping was performed on animals after treatment.

All animal experiments and care protocols were approved by the Home Office (PPL70/8784) and UCL Animal Welfare and Ethical Review Body. All procedures were carried out in accordance with the UK Animals (Scientific Procedures) Act 1986 and the ARRIVE guidelines. All experiments involved postnatal day 7 mice (P7) bred in house. At P7, the neonatal mouse brain development is comparable to a mid-third-trimester human fetus or newborn infant, with complete cortical neuronal layering, an involuted germinal matrix, and slightly myelinated white matter (24). Although slightly preterm, the murine P7 model of HI presents phenotypical similarities to the gray and white matter injury observed in humans, including tissue loss, cell-death, microglia-mediated immune response and astrogliosis as well as changes in neurological behavior (24).

Because properdin is located on the $\mathrm{X}$-chromosome, mating of heterozygous properdin deficient females with wild type males yields male hemizygous, properdin-deficient and wild type mice (as well as female heterozygous and wild type mice). Therefore, only male pups were used in the experiments and were ideally controlled as littermates. According to clinical and experimental evidence, male mice may express a worse phenotype post-HI than female mice, with increased loss of male hippocampal volume after chronic postnatal hypoxia (25). All the assessments were performed blindly to the genotype.

\section{HI Insult}

The surgical procedures, a variation of the Rice-Vannucci rodent HI model, were performed as previously described (7, 26-30). Briefly, a total of 30 P7 male mice, both wild type $(n=15)$ and with global properdin deletion $(n=15)$, were anesthetized using 
isoflurane (5\% induction, $1.5 \%$ maintenance). The left carotid artery was permanently occluded (8/0 propylene suture) and the wound was closed with tissue glue. The mice were left to recover at $36^{\circ} \mathrm{C}$ and returned to the dam for $2 \mathrm{~h}$. They were then moved to a hypoxia chamber and exposed to humidified $8 \%$ oxygen, $92 \%$ nitrogen $(3 \mathrm{~L} / \mathrm{min}$ ) for $60 \mathrm{~min}$ (HI alone) or $30 \mathrm{~min}$ (LPS-sensitized $\mathrm{HI}$ ) at $36^{\circ} \mathrm{C}(27)$, resulting in moderate to severe brain damage $(27,29,30)$. In the infection-sensitized HI insult, 55 P6 pups from both genotypes were injected with E. coli lipopolysaccharide (LPS; $0.6 \mu \mathrm{g} / \mathrm{g}$, serotype 055:B5; Fluka, UK) $(n=13 \mathrm{WT}, n=13 \mathrm{KO})$ or saline $12 \mathrm{~h}$ prior to surgery $(n=15$ $\mathrm{WT}, n=15 \mathrm{KO})(7,29)$. The contralateral side of the brain served as an intra-animal control reference for ipsilateral damage.

\section{Tissue Sample Preparation}

The animals were sacrificed at $48 \mathrm{~h}$ following the $\mathrm{HI}$ insult using intraperitoneally delivered pentobarbitone. They were perfused with $30 \mathrm{~mL} 4 \%$ paraformaldehyde (PFA) in phosphate-buffered saline (PBS). The brains were then extracted, post-fixed for $1 \mathrm{~h}$ in $4 \% \mathrm{PFA} / 0.1 \mathrm{M}$ phosphate buffer $(\mathrm{PB})$ at $4^{\circ} \mathrm{C}$, before being cryoprotected in $30 \%$ sucrose/PB solution for $24 \mathrm{~h}$. The cerebellum was removed from each brain. The forebrains were frozen on dry ice, cut into 50 sequential $40 \mu \mathrm{m}$ coronal sections starting from the fusion of corpus callosum, and the slices were stored at $-80^{\circ} \mathrm{C}(7,27,29,30)$.

\section{Immunohistochemistry and Histological Analysis}

Five sections from each brain $(400 \mu \mathrm{m}$ apart) were rehydrated in distilled water and stained using immunohistochemistry as previously described $(7,27,29,30)$. Briefly, the sections were incubated overnight with rat anti-CD11b $\alpha \mathrm{M}$ integrin subunit (1:5,000, Serotec, UK) or rabbit polyclonal anti-glial fibrillary acidic protein (GFAP) (1:6,000, DAKO, UK), primary antibodies, for $1 \mathrm{~h}$ with biotinylated goat anti-rat or -rabbit (1:100, Vector, UK) secondary antibodies, followed by incubation with AvidinBiotinylated horseradish peroxidase Complex (Vector, UK) and visualization with diaminobenzidine $/ \mathrm{H}_{2} \mathrm{O}_{2}$ (Fisher Scientific, UK) $(7,27,29,30)$.

Five further sections from each brain with the same spacing were stained using Terminal transferase mediated d-UTP nick end labeling (TUNEL) (Roche, UK). The staining procedure followed the manufacturer protocol with $\mathrm{Co} / \mathrm{Ni}$ enhancement $(7,27,29,30)$.

Five more sections per brain with the same spacing were stained with Cresyl-Violet (Nissl).

\section{AlphaM Score}

Assessment for $\alpha \mathrm{M}$ integrin immunoreactivity as a marker for early microglial activation (7, 26-30) was performed as previously described $(7,27,30)$. Two independent observers blinded to the genotype and treatment of the groups allocated semi-quantitative scores to each brain region, i.e., cortex, pyriform cortex, hippocampus, striatum, thalamus, and external capsule.

\section{Optical Luminosity}

The central cytoskeletal framework of astroglia comprises GFAP, a type III intermediate filament found only in glial cells in the CNS. GFAP upregulation is seen during HI-triggered reactive gliosis (31). In order to quantify the intensity of the GFAP staining, we used optical luminosity values (OLV) as a wellestablished technique $(7,26-30)$. Images for ipsilateral and contralateral sides were captured with a Sony AVT-Horn 3CCD color video camera ( 24 bit RGB, $760 \times 570$ pixel resolution) in three different optical fields in cortex, pyriform cortex, hippocampus, striatum, thalamus and external capsule. We used Optimas 6.5 software to obtain the mean and standard deviation (SD) for OLVs. SD was subtracted from the mean for each image, and the resulting value was subtracted from the values acquired for the surrounding glass.

\section{TUNEL Assessment}

As a measure of cell death at $48 \mathrm{~h}$ post-HI, the number of TUNEL + cells was counted in three different optical fields at $\times 20$ magnification. The cortex, pyriform cortex, hippocampus, striatum, thalamus and external capsule were assessed. The counts were averaged per animal and per group.

\section{Infarct Volume Measurement}

Cresyl violet dye stains Nissl bodies present in the cytoplasm of neurons. In this study we used Nissl stain to measure tissue loss in the cortex, pyriform cortex, hippocampus, striatum and thalamus. Nissl-stained brain sections were imaged with Sony AVT-Horn 3CCD color video camera (24 bit RGB, $760 \times 570$ pixel resolution) at $\times 1$ magnification. The images were imported in Fiji Image J (NIH, USA), and the areas of intact staining in all 6 regions were outlined and bilaterally measured. The percentage tissue loss was then calculated by converting the measured uninjured areas into square millimeters and then transformed to a volume through multiplication by $400 \mu \mathrm{m}$. The sum of these volumes was then used to calculate the percentage of surviving brain tissue as ipsilateral/contralateral $\times 100(32)$.

\section{Statistics}

GraphPad Prism 8 (La Jolla, CA, United States) and SPSS 25.0 (IBM, USA) were used to perform all statistical analyses. The same six forebrain regions (cortex, pyriform cortex, hippocampus, striatum, thalamus, external capsule) were used for each outcome and each assay.

As the data from the Rice-Vannucci model are mostly nonnormally distributed, we performed non-parametric MannWhitney test (HI alone set of experiments) to compare the effect of global properdin deletion in each brain region separately, and a two-tailed $p$-value was assumed. As the number of groups in the LPS-sensitized HI set of experiments was larger than two, we performed the non-parametric Kruskal-Wallis test followed by Bonferroni-corrected pairwise-contrasts. Alpha was set to 0.05 . All data are graphically presented as Median $\pm \mathrm{IQR}$ (interquartile range, presented as error bars). All hypotheses were two-tailed and all data illustrate the response in the ipsilateral (experimental) hemisphere. In our data, a main effect is the effect of an independent variable (treatment) on a dependent 
variable (damage marker) averaged across the levels of any other independent variables (brain regions).

\section{RESULTS}

\section{Global Properdin Deletion Reduces Cell Death and Microglial Activation Following Neonatal HI-Insult}

Global deletion of properdin significantly reduced brain damage markers (cell death and microglial activation) compared to wild type control animals at $48 \mathrm{~h}$ post-HI. As shown in Figures $1 \mathrm{~A}-\mathrm{C}$, global properdin deletion significantly reduced the number of TUNEL+ cells compared to wild type controls, with individual significant decrease of $20-38 \%$ in pyriform cortex, hippocampus, striatum, thalamus and overall ( $p<0.05$, Mann-Whitney test). The TUNEL+ cells displayed the typical pyknotic nuclear morphology and high density in the control group (Figure 1B-insert, ipsilateral hippocampus) compared to the reduced number of such cells in the properdin KO brains (Figure 1C-insert, ipsilateral hippocampus).

The regional assessment presented in Figure 1D revealed slight decrease of ipsilateral brain tissue volume loss in the pyriform cortex and thalamus in the global properdin deletion group (Figure 1F) compared to wild type controls (Figure 1E), however the data did not reach significant values.

Assessment of ipsilateral astrogliosis through GFAP immunoreactivity (Figure 1G) showed that compared to wild type controls (Figure 1H), global properdin deletion had no effect on reactive astrogliosis (Figure 1I) following neonatal HI.

In addition to cell death, global properdin deletion had a significant effect on ipsilateral microglia activation score (Figure 1J) based on $\alpha \mathrm{M}$ integrin immunoreactivity (Figures 1K,L). Regional assessment shown in Figure 1J revealed a reduction in activation score in the properdin $\mathrm{KO}$ group, with individual decrease of $21-76 \%$ in pyriform cortex, hippocampus, striatum, thalamus and overall ( $p<0.05$, Mann-Whitney test). At high magnification, the $\alpha \mathrm{M}+$ cells in the wild type control group showed phagocytic morphology (Figure 1K-insert, ipsilateral hippocampus) compared to the ramified phenotype of these cells observed in the animals with global properdin deletion (Figure 1L_-insert, ipsilateral hippocampus).

\section{Global Properdin Deletion Reduces Brain Damage Following LPS-Sensitized Neonatal HI-Insult}

Global deletion of properdin significantly reduced brain damage markers (cell death, tissue loss and microglial activation) compared to wild type control animals at $48 \mathrm{~h}$ post LPS-sensitized neonatal HI. As shown in Figures 2A-D, global properdin deletion significantly reduced the number of TUNEL+ cells compared to LPS-treated wild type controls (main effect, $p<$ 0.05 , Kruskal-Wallis test), with individual decrease of $50-76 \%$ in all 6 regions, but reaching significance in cortex, pyriform cortex, hippocampus and overall (Bonferroni correction, $p<0.05$ ). The saline-treated wild type controls showed very low number of TUNEL+ cells (Figures 2A,B), and global properdin deletion did not affect those numbers (Figure 2A). LPS-sensitization resulted in a substantial increase of TUNEL + cell death observed in the wild type LPS-treated group (Figure 2C) compared to saline treated wild types (Figure 2B). The TUNEL+ cells displayed the typical pyknotic nuclear morphology and high density in the LPS-treated wild type group (Figure 1C-insert, ipsilateral hippocampus) compared to the reduced number of such cells in the LPS-treated properdin KO brains (Figure 1D-insert, ipsilateral hippocampus).

Regional assessment presented in Figure 2E revealed very low levels of ipsilateral tissue loss in the saline-treated wild type animals (Figure 2F), and global properdin deletion did not affect these levels (Figure 2E). LPS-sensitization resulted in an extensive increase of tissue loss observed in the LPStreated wild type group (Figure 2G) compared to the saline treated wild type controls (Figure 2F). We observed an overall trend toward reduction of ipsilateral brain tissue volume loss of 13-66\% across all 6 forebrain regions in the LPStreated global properdin deletion group (Figure $2 \mathrm{H}$ ) compared to LPS-treated wild type controls (Figure 2G) (main effect, $p<0.05$, Kruskal-Wallis test), however the data reached significant values only in cortex and pyriform cortex (Bonferroni correction, $p<0.05$ ).

Similarly, regional assessment of ipsilateral astrogliosis through GFAP immunoreactivity (Figure 2I) showed very low levels of reactive astrogliosis in saline-treated wild type controls (Figure 2J), and global properdin deletion did not affect those levels (Figure 2I). LPS-sensitization resulted in a considerable increase of astroglial activation observed in the LPS-treated wild type group (Figure 2K) compared to the saline treated wild type controls (Figure 2J). We observed an overall trend toward reduction of ipsilateral reactive astrogliosis of $27 \%$ across all 6 forebrain regions in the LPS-treated global properdin deletion group (Figure 2L) compared to LPStreated wild type controls (Figure 2K) (main effect, $p<$ 0.05 , Kruskal-Wallis test), however the data did not reach significant values.

Additionally, assessment of microglial activation based on $\alpha \mathrm{M}$ integrin immunoreactivity (Figure $\mathbf{2 M}$ ) showed very low levels of $\alpha \mathrm{M}+$ microglia in saline-treated wild type controls (Figure 2N), and global properdin deletion did not affect those levels (Figure 2M). LPS-sensitization resulted in a substantial increase of microglial activation observed in the LPS-treated wild type group (Figure 2O) compared to the saline treated wild type controls (Figure 2M). Regional assessment shown in Figure 2M revealed a reduction in activation score of 31$66 \%$ in all 6 individual ipsilateral brain regions in the LPStreated global properdin deletion group compared to the LPStreated wild type controls (main effect, $p<0.05$, Kruskal-Wallis test), however significance was reached only in hippocampus (Bonferroni correction, $p<0.05$ ). At high magnification the $\alpha \mathrm{M}+$ cells in the LPS-treated wild type control group showed phagocytic morphology and high density (Figure 2O-insert, ipsilateral hippocampus) compared to the ramified resting phenotype and low density of these cells observed in the LPStreated global properdin deletion animals (Figure $\mathbf{2 P}$-insert, ipsilateral hippocampus). 


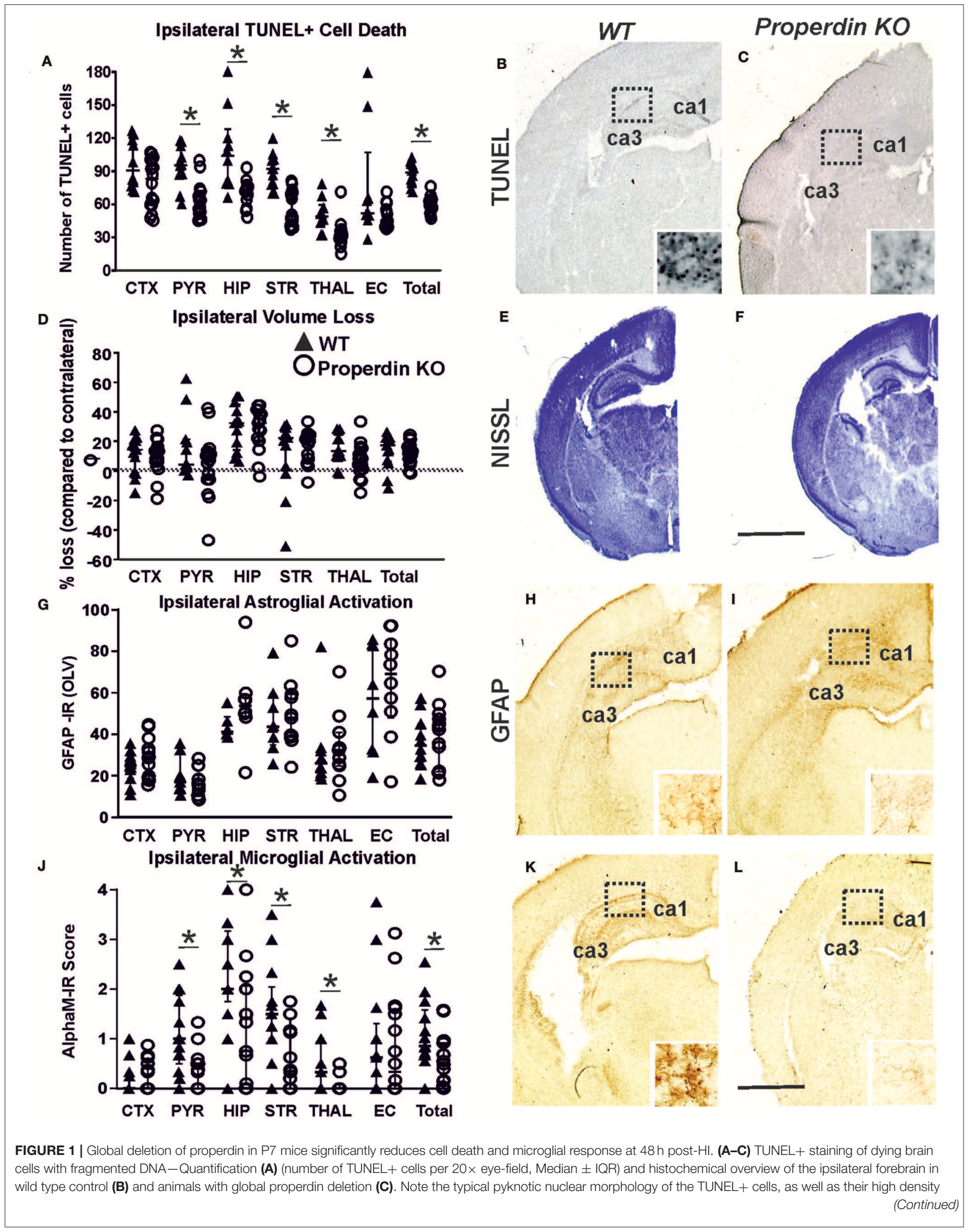


FIGURE 1 | observed in the controls (B-insert, hippocampus) compared to the reduced number of such cells in the properdin KO group (C-insert, hippocampus). Compared to wild type controls, properdin deletion resulted in reduced TUNEL+ cell death across all 6 examined forebrain regions, with significant, individual decrease (Mann-Whitney test) in the pyriform cortex $(p=0.0004)$, hippocampus $(p=0.0008)$, striatum $(p<0.0001)$, thalamus $(p=0.0034)$ and overall $(p<0.0001$ ). (D-F) Ipsilateral forebrain Nissl staining (Cresyl-Violet, at rostral parietal level) -Quantification of percentage of ipsilateral brain tissue volume loss (D, Median \pm IQR) of wild type control $(\mathbf{E})$ and properdin $\mathrm{KO}(\mathbf{F})$ animals. Compared to wild type controls global properdin deletion resulted in slight decrease of volume loss following neonatal $\mathrm{HI}$, however the data did not reach significant values. (G-I) GFAP immunoreactivity-Quantification of ipsilateral reactive astrogliosis (G) in optical luminosity values (OLV, Median \pm IQR), and low magnification ipsilateral overview in wild type control $\mathbf{( H )}$ and animals with global properdin deletion (I). The inserts in $\mathrm{H}$ and I show higher magnification of the dotted regions in hippocampus. Global properdin deletion did not have an effect on astroglial activation following neonatal HI. (J-L) Activation of $\alpha \mathrm{M}+$ microglia-Ipsilateral $\alpha \mathrm{M}$ microglial activation score ( $\mathbf{J}$, Median $\pm \mathrm{IQR}$ ) and low magnification ipsilateral overview in wild type control (K) and animals with global properdin deletion (L). Note the strong microglial activation in the control wild type group with aM+ cells showing phagocytic morphology at high magnification (K-insert, hippocampus), compared to the properdin KO brains exhibiting a ramified phenotype ( $\mathbf{L}-$ insert). Global properdin deletion reduced $\alpha \mathbf{M}+$ microglial activation across all 6 examined forebrain regions apart from cortex, with significant, individual decrease (Mann-Whitney test) in pyriform cortex $(p=0.008)$, hippocampus $(p=0.05)$, striatum $(p=0.02)$, thalamus $(p=0.04)$, and overall $(p=0.01)$. Wild type $(n=14)$ and global properdin deletion $(n=16)$ in all assessments. $\left({ }^{*} P<0.05\right)$. CTX, cerebral cortex; PYR, pyriform cortex; HIP, hippocampus; STR, striatum; THAL, thalamus; EC, external capsule. Scale bars: $(\mathbf{E}, \mathbf{F})=1,200 \mu \mathrm{m}$; $\mathbf{( B , C , H , I , K , L )}=600 \mu \mathrm{m}$. inserts $=30 \mu \mathrm{m}$.

\section{DISCUSSION}

Lack of oxygen to the fetal brain around the time of birth is a major cause of neonatal HI brain damage, triggering neurological sequelae such as cerebral palsy, epilepsy and mental retardation. Intrauterine or perinatal complications, including maternal infection, constitute a major risk for the development of neonatal HI brain damage. The mechanisms underlying the trigger of brain damage under the conditions of $\mathrm{HI}$ alone and LPS-sensitized HI overlap, but also differ $(7,29)$. Therefore, our study aimed to validate the effect of global properdin deletion in two models: HI alone and LPS-sensitized HI, thus addressing two different clinical scenarios.

In the current study, C57/Bl6 background was chosen as a result of the high severity of $\mathrm{HI}$ injury incurred following prolonged hypoxic exposure $(7,33)$.

Additionally, global properdin deletion reduced forebrain cell death and microglial activation, as well as tissue loss in a Rice-Vannucci model of neonatal HI and LPS-sensitized HI brain damage.

In the model of $\mathrm{HI}$ alone, deletion of properdin reduced brain damage based on evidence for TUNEL + cell death and microglial post-HI response, which in both assessments reached significance.

Under the conditions of LPS-sensitized HI, properdin deletion reduced brain injury based on evidence of significantly diminished cell death, tissue loss and post-HI microglial activation. Overall, our data suggests a critical role for properdin, and possibly also a contribution in neonatal $\mathrm{HI}$ alone, as well as in infection-sensitized HI brain damage.

Complement is an essential part of innate immunity and participates not only in normal brain physiology, but also under pathological conditions, including ischemia (34). Absence of the $\mathrm{CP}$ in the neonatal HI brain is neuroprotective $(35,36)$. Despite the long history of research on the role of complement in neonatal $\mathrm{HI}(35,36)$, there is a paucity of data on the participation of the AP in post-HI brain damage (34), in particular the role of properdin.

Our data shows that in HI alone and in LPS-sensitized HI, properdin deficiency reduced TUNEL+ cell death with significant differences in pyriform cortex, hippocampus, striatum, thalamus and overall in the $\mathrm{HI}$ alone set of experiments (Figure 1A), and in cortex, pyriform cortex and hippocampus in the LPS-sensitized HI (Figure 2A). Additionally, tissue loss was significantly reduced in the thalamus in the $\mathrm{HI}$ alone set of experiments (Figure 1D) and in the cortex and pyriform cortex in the LPS-sensitized HI (Figure 2B). Sub-regional differences in the vulnerability of the different brain regions to HI damage exist based on metabolic rate and energy demand (6). HI insult around term, as modeled in this study, damages predominantly gray matter in the cortex, hippocampus and/or thalamus (37). In animal models, damage in the cortex, thalamus and striatum has been associated with sensorimotor impairment (38-41). Interestingly, as the hippocampus is one of the regions with the highest metabolic rate in the developing brain and therefore highly susceptible to HI injury, damage to it and to the cortico-hippocampal projections causes memory and spatial processing dysfunction (42). Additionally, HI-induced reduction in hippocampal volume has been associated with impaired long-term reference memory, short-term working memory (43), as well as spatial navigation and recollection (44, 45). We observed reduced cell death in striatum of animals with global properdin deletion following neonatal HI (Figure 1A). As damage to the striatum, in particular to nucleus accumbens may have an impact on non-spatial navigation and learning $(45,46)$ and might explain non-spatial memory deficits in neonatal HI rats (37), protection of that region is essential. Neonatal HI is considered a major risk factor for psychiatric diseases including attention-deficit hyperactivity disorder (ADHD), autism, psychosis and schizophrenia (47-51). The main regions associated with related cognitive functions are the hippocampus and striatum as well as cortico-hippocampal and cortico-striatal projections. As global properdin deletion provides neuroprotection for these regions, it is likely that it would reduce the risk of development of later life psychological and behavioral complications, however that would require additional long-term behavioral studies.

Although there is no data on the effect of global properdin deletion on neonatal HI brain damage, our results are in line with previous studies looking at the role of the AP in murine models of stroke. In an adult mouse study of middle cerebral artery occlusion (MCAO), C3 deficiency and site-targeted inhibition 


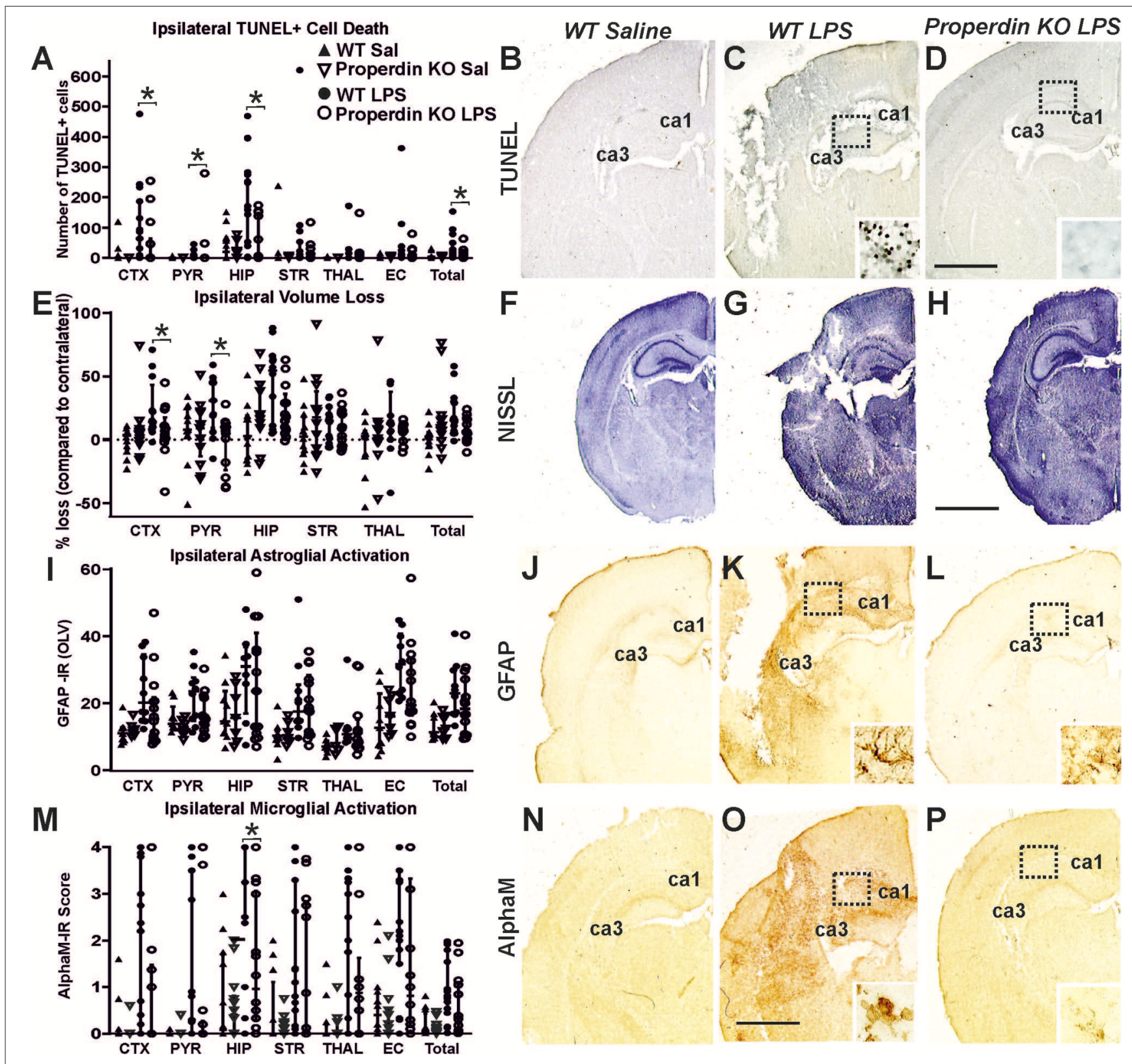

FIGURE 2 | Global deletion of properdin in P7 mice significantly reduces cell death tissue loss and microglial response at 48 h post LPS-sensitized HI. (A-D) TUNEL+ staining of dying brain cells with fragmented DNA-Quantification (A) (number of TUNEL+ cells per 20x eye-field, Median \pm IQR) and histochemical overview of the ipsilateral forebrain in saline-treated wild type (B), LPS-treated wild type controls (C) and LPS-treated animals with global properdin deletion (D). The saline-treated wild type animals showed a very low number of TUNEL+ cells (B). Note the typical pyknotic nuclear morphology of the TUNEL+ cells as well as their high density observed in the LPS-treated wild type controls (C-insert, hippocampus) compared to the reduced number of such cells in the LPS-treated properdin KO group (D-insert, hippocampus). Compared to the wild type controls, properdin deletion resulted in reduced TUNEL+ cell death with a significant main effect (Kruskal-Wallis test, $p=0.007$ ), and significant, individual decrease (Bonferroni correction) in cortex $(p=0.04)$, pyriform cortex $(p=0.03)$, hippocampus ( $p=0.02$ ) and overall $(p=0.05)$. (E-H) Ipsilateral forebrain Nissl staining (Cresyl-Violet, at rostral parietal level, Median \pm IQR)-Quantification of ipsilateral brain tissue volume loss (E) of saline-treated wild type (F), LPS-treated wild type controls (G), and LPS-treated animals with global properdin deletion $\mathbf{( H )}$. The saline-treated wild type animals showed very low levels of ipsilateral tissue loss (F). Compared to wild type controls, global properdin deletion resulted in a decrease of volume loss following LPS-sensitized neonatal HI with a significant main effect (Kruskal-Wallis test, $p=0.0001$ ) and significant individual decrease (Bonferroni correction) in cortex $(p=0.044)$ and pyriform cortex $(p=0.009)$. (I-L) GFAP immunoreactivity - Quantification of ipsilateral reactive astrogliosis (G) in optical luminosity values (OLV, Median \pm IQR) and low magnification ipsilateral overview in saline-treated wild type (J), LPS-treated wild type controls (K) and LPS-treated animals with global properdin deletion (L). The saline-treated wild type animals showed very low levels of ipsilateral GFAP immunoreactivity (J). The inserts (K,L) show higher magnification of the dotted regions in hippocampus. Compared to wild type controls, global properdin deletion decreased reactive astrogliosis with a significant main effect (Kruskal-Wallis test, $p=0.0001$ ), however the sub-regional differences did not reach significant values. (M-P) Activation of $\alpha \mathbf{M}+$ microglia-lpsilateral $\alpha \mathbf{M}$ microglial activation score 
FIGURE 2 | (M, Median \pm IQR) and low magnification ipsilateral overview in saline-treated wild type (N), LPS-treated wild type controls (O), and LPS-treated animals with global properdin deletion (P). The saline-treated wild type controls showed very low levels of $a \mathrm{M}+$ microglia (N). Note the strong microglial activation in the LPS-treated wild type control group with aM+ cells showing phagocytic morphology at high magnification ( $\mathbf{O}$-insert, hippocampus), compared to the LPS-treated properdin KO brains exhibiting a ramified phenotype ( $\mathbf{P}$-insert, hippocampus). Global properdin deletion reduced $\alpha \mathrm{M}+$ microglial activation with a significant main effect (Kruskal-Wallis test, $p=0.0001$ ) and significant, individual decrease (Bonferroni correction) in hippocampus $(p=0.05)$. Saline wild type ( $n=12)$, saline properdin KO $(n=14)$, LPS-treated wild type $(n=15)$ and LPS-treated properdin $\mathrm{KO}(n=14)$ in all assessments. $\left({ }^{*} p<0.05\right)$. CTX, cerebral cortex; PYR, pyriform cortex; HIP, hippocampus; STR, striatum; THAL, thalamus; EC, external capsule. Scale bars: (F-H) =1,200 $\mu \mathrm{m}$; (B-D,J-L,N,0), $p=600 \mu \mathrm{m}$. inserts $=30 \mu \mathrm{m}$.

with either CR2-Crry (inhibiting all pathways) or CR2-fH (inhibiting AP) significantly reduced infarct size, reduced apoptotic cell death, and improved neurological deficit score in the acute phase after stroke, but only CR2-fH provided sustained protection with no further development of injury in the subacute phase (52). Similarly, Ten et al. (36) demonstrated that $\mathrm{C} 3$ deficiency provided protection against $\mathrm{MCAO}$ as well as against neonatal HI. Additionally, intranasal C3a treatment ameliorated cognitive impairment in a mouse model of $\mathrm{HI}$ brain injury (35). However, C3 deficiency takes away the component central to all three complement pathways compared to properdin deficiency, which reduces these activities. Similarly, factor B-deficiency or CR2-fH treatment improved neurological function and reduced cerebral infarct, demyelination, P-selectin expression and neutrophil infiltration following MCAO in adult mice (52). Although the model of injury in MCAO is technically different than neonatal $\mathrm{HI}$, both models share similarities, including oxygen deprivation and reperfusion, thus effects observed in MCAO could be plausible in neonatal $\mathrm{HI}$ and vice versa.

Our results show reduced microglial activation in all studied regions apart from cortex in the properdin deficient group following neonatal HI alone (Figure 1J). Similarly, in the LPS-sensitized HI model, we observed a main effect of the global properdin deletion with significant reduction observed in hippocampus (Figure 2M). This suggests reduced inflammatory response and subsequent cell death (Figures 1A, 2A). Inflammation plays a major part in the pathology of neonatal $\mathrm{HI}$ brain damage $(6,34,53)$. Cerebral ischemia induces inflammation in both systemic circulation and the parenchyma. In an adult brain, this results in increased production of cytokines, as well as activation and migration of leukocytes to the injured brain $(34,53)$. In neonates, however, the result is an immediate innate immune response following the insult. The differences in the mechanisms between adult stroke and neonatal $\mathrm{HI}$ are mostly due to the immaturity of the neonatal CNS, resulting in insufficient ability to overcome excitotoxicity, oxidative stress and inflammation. HI damage is suggested to occur because of imbalance between pro- and anti-inflammatory cytokines, which boosts oligodendrocyte precursors to proliferate into astrocytes instead of oligodendrocytes, thus impairing subsequent myelination (54). In some models, presence of properdin has been associated with increased production of pro-inflammatory cytokines (TNF-alpha, IL-1b, and IL-6) and suppressed levels of anti-inflammatory cytokines (IL-10 and $\mathrm{TGFb}$ ) (55). Therefore, its presence following HI might be a contributing factor for the imbalance between pro- and anti-inflammatory cytokines. We have previously shown that inhibition of IL-6 downstream products such as phosphorylated STAT3 is neuroprotective in neonatal HI (27). Hence, the lack of properdin would prevent IL-6 upregulation and provide neuroprotection in neonatal HI. Therefore, it can be assumed that deletion of properdin would exhibit a neuroprotective effect through reduction of pro-inflammatory cytokine levels, thus preserving the equilibrium between pro- and anti-inflammatory cytokines and ensuring subsequent myelination. Additionally, properdin is required for the AP activation when LPS is present (56). Thus, deficiency in properdin in the presence of LPS would prevent AP activation and ensuing inflammatory response. In addition to the increase of pro-inflammatory cytokines triggered by HI alone, LPS causes further upregulation of TNF-alpha, IL-1b, and IL-6. As properdin deletion might interfere with the execution of IL-6 dependent inflammatory response, it is possible that inherited properdin deficiency inhibits LPS sensitivity in neonates. Conversely, in a study looking at zymosan-induced and LPS-induced septic shock in adult mice, properdin deletion provides protection only in the case of zymosan-, but not in LPS-induced septic shock (57). However, the model of septic shock involves different mechanisms which can explain the differences in the effects of global properdin deletion.

Our data did not support an effect of properdin deletion on astroglial activation in HI alone or in LPS-sensitized HI, suggesting that the protective role of properdin in both models is likely due to impairment of the microglia-dependent proinflammatory response post-HI.

As a conclusion, our study provides evidence that properdin is involved and likely plays a key role in the trigger of neonatal HI and LPS-sensitized HI brain damage. Although our study was limited to male gender and the $\mathrm{HI}$ insult in the $\mathrm{HI}$ alone set of experiments was moderate rather than severe, global properdin deletion provides neuroprotection in the short term $(48 \mathrm{~h})$ in both models on the grounds of reduced cell death, tissue loss and microglial activation. The likely mechanism underlying these protective effects is impairment of the microglial proinflammatory response, which would prevent imbalance between pro- and anti-inflammatory cytokines following $\mathrm{HI}$ insult and would preserve subsequent myelination, however that would require further investigation. Overall our data suggest properdin as a novel target for treatment in neonatal HI brain damage; however, a better understanding of the pathway(s) through which it is involved in HI-brain damage would considerably improve the therapeutic potential of interfering with it in a clinical setting. 


\section{DATA AVAILABILITY STATEMENT}

All datasets generated for this study are included in the article/supplementary material.

\section{ETHICS STATEMENT}

This study was carried out in accordance with the UK Animals (Scientific Procedures) Act 1986 and the ARRIVE guidelines. The protocols were approved by the Home Office (PPL70/8784) and UCL Animal Welfare and Ethical Review Body.

\section{REFERENCES}

1. Kurinczuk JJ, White-Koning M, Badawi N. Epidemiology of neonatal encephalopathy and hypoxic-ischemic encephalopathy. Early Hum Dev. (2010) 86:329-38. doi: 10.1016/j.earlhumdev.2010.05.010

2. Lai M-C, Yang S-N. Perinatal hypoxic-ischemic encephalopathy. J Biomed Biotechnol. (2011) 2011:609813. doi: 10.1155/2011/609813

3. Gluckman PD, Williams CE. When and why do brain cells die? Dev Med Child Neurol. (1992) 34:1010-4. doi: 10.1111/j.1469-8749.1992.tb11407.x

4. Lorek A, Takei Y, Cady EB, Wyatt JS, Penrice J, Edwards AD, et al. Delayed ("secondary") cerebral energy failure after acute hypoxia-ischemia in the newborn piglet: continuous 48-hour studies by phosphorus magnetic resonance spectroscopy. Pediatr Res. (1994) 36:699-706. doi: 10.1203/00006450-199412000-00003

5. Penrice J, Cady EB, Lorek A, Wylezinska M, Amess PN, Aldridge RF, et al. Proton magnetic resonance spectroscopy of the brain in normal preterm and term infants, and early changes after perinatal hypoxia-ischemia. Pediatr Res. (1996) 40:6-14. doi: 10.1203/00006450-199607000-00002

6. Rocha-Ferreira E, Hristova M. Plasticity in the neonatal brain following hypoxic-ischemic injury. Neural Plast. (2016) 2016:1-16. doi: 10.1155/2016/4901014

7. Rocha-Ferreira E, Phillips E, Francesch-Domenech E, Thei L, Peebles DM, Raivich G, et al. The role of different strain backgrounds in bacterial endotoxin-mediated sensitization to neonatal hypoxic-ischemic brain damage. Neuroscience. (2015) 311:292-307. doi: 10.1016/j.neuroscience.2015.10.035

8. Wang X, Stridh L, Li W, Dean J, Elmgren A, Gan L, et al. Lipopolysaccharide sensitizes neonatal hypoxic-ischemic brain injury in a MyD88-dependent manner. J Immunol. (2009) 183:7471-7. doi: 10.4049/jimmunol.0900762

9. Azzopardi D, Strohm B, Linsell L, Hobson A, Juszczak E, Kurinczuk JJ, et al. Implementation and conduct of therapeutic hypothermia for perinatal asphyxial encephalopathy in the UK-Analysis of national data. PLOS ONE. (2012) 7:e38504. doi: 10.1371/journal.pone.0038504

10. Falck M, Osredkar D, Maes E, Flatebø T, Wood TR, Walløe L, et al. Hypothermia is neuroprotective after severe hypoxic-ischemic brain injury in neonatal rats pre-exposed to PAM3CSK4. Dev Neurosci. (2018) 40:189-97. doi: $10.1159 / 000487798$

11. Osredkar D, Thoresen M, Maes E, Flatebø T, Elstad M, Sabir H. Hypothermia is not neuroprotective after infection-sensitized neonatal hypoxic-ischemic brain injury. Resuscitation. (2014) 85:567-72. doi: 10.1016/j.resuscitation.2013.12.006

12. Wintermark $P$, Boyd T, Gregas MC, Labrecque M, Hansen A. Placental pathology in asphyxiated newborns meeting the criteria for therapeutic hypothermia. Am J Obstet Gynecol. (2010) 203:579.e1-579.e9. doi: 10.1016/j.ajog.2010.08.024

13. Chalak LF, Sánchez PJ, Adams-Huet B, Laptook AR, Heyne RJ, Rosenfeld CR. Biomarkers for severity of neonatal hypoxic-ischemic encephalopathy and outcomes in newborns receiving hypothermia therapy. J Pediatr. (2014) 164:468-474.e1. doi: 10.1016/j.jpeds.2013.10.067

14. Nakamura T, Yamada S, Yoshioka T. Brain hypothermic therapy dramatically decreases elevated blood concentrations of high mobility group box 1 in

\section{AUTHOR CONTRIBUTIONS}

CSi: collection and processing of data, writing, and editing the manuscript. QA-S, BS, and AC: collection and processing of data. CSt: provision of the properdin deficient animals, independent genotyping, writing, and editing the manuscript. $\mathrm{MH}$ : design of the study, processing of data, writing, and editing the manuscript.

\section{FUNDING}

This work was supported by the BBSRC LIDo programme BB/M009513/1.

neonates with hypoxic-ischemic encephalopathy. Dis Markers. (2013) 35:32730. doi: 10.1155/2013/327604

15. Elvington A, Atkinson C, Zhu H, Yu J, Takahashi K, Stahl GL, et al. The alternative complement pathway propagates inflammation and injury in murine ischemic stroke. J Immunol. (2012) 189:4640-7. doi: 10.4049/jimmunol.1201904

16. Lesher AM, Nilsson B, Song W-C. Properdin in complement activation and tissue injury. Mol. Immunol. (2013) 56:191-8. doi: 10.1016/j.molimm.2013.06.002

17. Hourcade DE. The role of properdin in the assembly of the alternative pathway C3 convertases of complement. J Biol Chem. (2006) 281:2128-32. doi: 10.1074/jbc.M508928200

18. Maves KK, Weiler JM. Properdin: approaching four decades of research. Immunol Res. (1993) 12:233. doi: 10.1007/BF02918255

19. Grether JK, Nelson KB, Dambrosia JM, Phillips TM. Interferons and cerebral palsy. J Pediatr. (1999) 134:324-32. doi: 10.1016/S0022-3476(99)70458-0

20. Sonntag J, Wagner MH, Strauss E, Obladen M. Complement and contact activation in term neonates after fetal acidosis. Arch Dis Child Fetal Neonatal Ed. (1998) 78:F125-8. doi: 10.1136/fn.78.2.F125

21. Kimura Y, Zhou L, Miwa T, Song WC. Genetic and therapeutic targeting of properdin in mice prevents complement-mediated tissue injury. J Clin Invest. (2010) 120:3545-54. doi: 10.1172/JCI41782

22. Miwa T, Sato S, Gullipalli D, Nangaku M, Song W-C. Blocking properdin, the alternative pathway and anaphylatoxin receptors ameliorates renal ischemia reperfusion injury in decay-accelerating factor and CD59 double knockout mice(). J Immunol. (2013) 190:3552-9. doi: 10.4049/jimmunol.1202275

23. Stover CM, Luckett JC, Echtenacher B, Dupont A, Figgitt SE, Brown J, et al. Properdin plays a protective role in polymicrobial septic peritonitis. $J$ Immunol. (2008) 180:3313-8. doi: 10.4049/jimmunol.180.5.3313

24. Vannucci RC, Vannucci SJ. A model of perinatal hypoxicischemic brain damage. Ann N Y Acad Sci. (1997) 835:234-49. doi: 10.1111/j.1749-6632.1997.tb48634.x

25. Mayoral SR, Omar G, Penn AA. Sex differences in a hypoxia model of preterm brain damage. Pediatr Res. (2009) 66:248-53. doi: 10.1203/PDR.0b013e3181b1bc34

26. Hristova M, Cuthill D, Zbarsky V, Acosta-Saltos A, Wallace A, Blight K, et al. Activation and deactivation of periventricular white matter phagocytes during postnatal mouse development. Glia. (2010) 58:11-28. doi: 10.1002/glia.20896

27. Hristova M, Rocha-Ferreira E, Fontana X, Thei L, Buckle R, Christou M, et al. Inhibition of Signal Transducer and Activator of Transcription 3 (STAT3) reduces neonatal hypoxic-ischemic brain damage. J Neurochem. (2016) 136:981-94. doi: 10.1111/jnc.13490

28. Kendall GS, Hristova M, Zbarsky V, Clements A, Peebles DM, Robertson NJ, et al. Distribution of $\mathrm{pH}$ changes in mouse neonatal hypoxic-ischemic insult. Dev Neurosci. (2012) 33:505-18. doi: 10.1159/000333850

29. Lange S, Rocha-Ferreira E, Thei L, Mawjee P, Bennett K, Thompson PR, et al. Peptidylarginine deiminases: novel drug targets for prevention of neuronal damage following hypoxic ischemic insult (HI) in neonates. J Neurochem. (2014) 130:555-62. doi: 10.1111/jnc.12744

30. Sisa C, Kholia S, Naylor J, Herrera Sanchez MB, Bruno S, Deregibus $\mathrm{MC}$, et al. Mesenchymal stromal cell derived extracellular vesicles reduce 
hypoxia-ischemia induced perinatal brain injury. Front Physiol. (2019) 10:282. doi: $10.3389 /$ fphys.2019.00282

31. Mondello S, Hayes RL. Chapter 16-Biomarkers. In: Grafman J, Salazar A, editors. Traumatic Brain Injury, Part I. Oxford: Elsevier (2015) 245-65. doi: 10.1016/B978-0-444-52892-6.00016-7

32. Kendall GS, Robertson NJ, Iwata O, Peebles D, Raivich G. N-methylisobutyl-amiloride ameliorates brain injury when commenced before hypoxia ischemia in neonatal mice. Pediatr Res. (2006) 59:227-31. doi: 10.1203/01.pdr.0000196805.68082.22

33. Sheldon RA, Sedik C, Ferriero DM. Strain-related brain injury in neonatal mice subjected to hypoxia-ischemia. Brain Res. (1998) 810:114-22. doi: 10.1016/S0006-8993(98)00892-0

34. Rocha-Ferreira E, Hristova M. Antimicrobial peptides and complement in neonatal hypoxia-ischemia induced brain damage. Front Immunol. (2015) 6:56. doi: 10.3389/fimmu.2015.00056

35. Morán J, Stokowska A, Walker FR, Mallard C, Hagberg H, Pekna M. Intranasal C3a treatment ameliorates cognitive impairment in a mouse model of neonatal hypoxic-ischemic brain injury. Exp Neurol. (2017) 290:74-84. doi: 10.1016/j.expneurol.2017.01.001

36. Ten VS, Sosunov SA, Mazer SP, Stark RI, Caspersen C, Sughrue $\mathrm{ME}$, et al. C1q-deficiency is neuroprotective against hypoxicischemic brain injury in neonatal mice. Stroke. (2005) 36:2244-50. doi: 10.1161/01.STR.0000182237.20807.d0

37. Alexander M, Garbus H, Smith AL, Rosenkrantz TS, Fitch RH. Behavioral and histological outcomes following neonatal $\mathrm{HI}$ injury in a preterm (P3) and term (P7) rodent model. Behav Brain Res. (2014) 259:85-96. doi: 10.1016/j.bbr.2013.10.038

38. Lubics A, Reglodi D, Tamás A, Kiss P, Szalai M, Szalontay L, et al. Neurological reflexes and early motor behavior in rats subjected to neonatal hypoxic-ischemic injury. Behav Brain Res. (2005) 157:157-65. doi: 10.1016/j.bbr.2004.06.019

39. Martinez-Biarge M, Diez-Sebastian J, Kapellou O, Gindner D, Allsop JM, Rutherford MA, et al. Predicting motor outcome and death in term hypoxic-ischemic encephalopathy. Neurology. (2011) 76:2055-61. doi: 10.1212/WNL.0b013e31821f442d

40. Mercuri E, Barnett A, Rutherford M, Guzzetta A, Haataja L, Cioni G, et al. Neonatal cerebral infarction and neuromotor outcome at school age. Pediatrics. (2003) 113:95-100. doi: 10.1542/peds.113.1.95

41. Mercuri E, Barnett AL. Neonatal brain MRI and motor outcome at school age in children with neonatal encephalopathy: a review of personal experience. Neural Plast. (2003) 10:51-7. doi: 10.1155/NP.2003.51

42. Aylward GP. Neurodevelopmental outcomes of infants born prematurely. J Dev Behav Pediatr. (2014) 26:427-40. doi: 10.1097/00004703-200512000-00008

43. Ikeda T, Mishima K, Yoshikawa T, Iwasaki K, Fujiwara M, Xia YX, et al. Selective and long-term learning impairment following neonatal hypoxic-ischemic brain insult in rats. Behav Brain Res. (2001) 118:17-25. doi: 10.1016/S0166-4328(00)00287-4

44. Packard MG, McGaugh JL. Inactivation of hippocampus or caudate nucleus with lidocaine differentially affects expression of place and response learning. Neurobiol Learn Mem. (1996) 65:65-72. doi: 10.1006/nlme.1996.0007

45. White NM, McDonald RJ. Multiple parallel memory systems in the brain of the rat. Neurobiol Learn Mem. (2002) 77:125-84. doi: 10.1006/nlme.2001.4008
46. Packard MG, Knowlton BJ. Learning and memory functions of the basal ganglia. Annu Rev Neurosci. (2002) 25:563-93. doi: 10.1146/annurev.neuro.25.112701.142937

47. De Haan M, Wyatt JS, Roth S, Vargha-Khadem F, Gadian D, Mishkin M. Brain and cognitive-behavioural development after asphyxia at term birth. Dev Sci. (2006) 9:350-8. doi: 10.1111/j.1467-7687.2006.00499.x

48. DeLong GR. Autism, amnesia, hippocampus, and learning. Neurosci Biobehav Rev. (1992) 16:63-70. doi: 10.1016/S0149-7634(05)80052-1

49. Hefter D, Marti HH, Gass P, Inta D. Perinatal hypoxia and ischemia in animal models of schizophrenia. Front Psychiatry. (2018) 9:106. doi: $10.3389 /$ fpsyt.2018.00106

50. Lou HC. Etiology and pathogenesis of Attention-deficit Hyperactivity Disorder (ADHD): Significance of prematurity and perinatal hypoxichaemodynamic encephalopathy. Acta Paediatr Int J Paediatr. (1996) 85:126671. doi: 10.1111/j.1651-2227.1996.tb13909.x

51. Van Petten C. Relationship between hippocampal volume and memory ability in healthy individuals across the lifespan: review and meta-analysis. Neuropsychologia. (2004) 42:1394-413. doi: 10.1016/j.neuropsychologia.2004.04.006

52. Alawieh A, Elvington A, Zhu H, Yu J, Kindy MS, Atkinson C, et al. Modulation of post-stroke degenerative and regenerative processes and subacute protection by site-targeted inhibition of the alternative pathway of complement. J Neuroinflamm. (2015) 12:247. doi: 10.1186/s12974-015-0464-8

53. Liu F, Mccullough LD. Inflammatory responses in hypoxic ischemic encephalopathy. Acta Pharmacol Sin. (2013) 34:1121-30. doi: 10.1038/aps.2013.89

54. Bain JM, Ziegler A, Yang Z, Levison SW, Sen E. TGFbeta1 stimulates the overproduction of white matter astrocytes from precursors of the "brain marrow" in a rodent model of neonatal encephalopathy. PLoS ONE. (2010) 5:e9567. doi: 10.1371/journal.pone.0009567

55. Al-Mozaini MA, Tsolaki AG, Abdul-Aziz M, Abozaid SM, Al-Ahdal MN, Pathan AA, et al. Human properdin modulates macrophage: mycobacterium bovis bcg interaction via thrombospondin repeats 4 and 5. Front Immunol. (2018) 9:533. doi: 10.3389/fimmu.2018.00533

56. Kimura Y, Miwa T, Zhou L, Song WC. Activator-specific requirement of properdin in the initiation and amplification of the alternative pathway complement. Blood. (2008) 111:732-40. doi: 10.1182/blood-2007-05-089821

57. Ivanovska ND, Dimitrova PA, Luckett JC, El-Rachkidy Lonnen R, Schwaeble WJ, Stover CM. Properdin deficiency in murine models of nonseptic shock. J Immunol. (2008) 180:6962-9. doi: 10.4049/jimmunol.180. 10.6962

Conflict of Interest: The authors declare that the research was conducted in the absence of any commercial or financial relationships that could be construed as a potential conflict of interest.

Copyright (c) 2019 Sisa, Agha-Shah, Sanghera, Carno, Stover and Hristova. This is an open-access article distributed under the terms of the Creative Commons Attribution License (CC BY). The use, distribution or reproduction in other forums is permitted, provided the original author(s) and the copyright owner(s) are credited and that the original publication in this journal is cited, in accordance with accepted academic practice. No use, distribution or reproduction is permitted which does not comply with these terms. 